\section{CVIA}

REVIEW ARTICLE

pISSN 2508-707X / eISSN 2508-7088 https://doi.org/10.22468/cvia.2018.00059 CVIA 2018;2(2):97-106
Received: March 14, 2018

Accepted: March 28, 2018

Corresponding author

Seitaro Oda, MD, PhD

Department of Diagnostic Radiology,

Faculty of Life Sciences,

Kumamoto University, 1-1-1, Honjo,

Chuo-ku, Kumamoto 860-8556, Japan

Tel: 81-96-373-5261

Fax: 81-96-373-5342

E-mail: seisei0430@nifty.com

\title{
Role of Noninvasive Diagnostic Imaging in Cardiac Amyloidosis: A Review
}

\author{
Seitaro Oda', Daisuke Utsunomiya', Takeshi Nakaura', \\ Seiji Takashio², Yasuhiro Izumiya², Kenichi Tsujita², \\ Mitsuharu Ueda ${ }^{3}$, Taro Yamashita ${ }^{3}$, Yukio Ando ${ }^{3}$, Yawara Kawano ${ }^{4}$, \\ Yutaka Okuno ${ }^{4}$, Hiroyuki Hata ${ }^{5}$, Yasuyuki Yamashita' \\ ${ }^{1}$ Departments of Diagnostic Radiology, ${ }^{2}$ Cardiology, ${ }^{3}$ Neurology, ${ }^{4}$ Hematology, Rheumatology, and \\ Infectious Disease, ${ }^{5}$ Informative Clinical Sciences, Faculty of Life Sciences, Kumamoto University, \\ Kumamoto, Japan
}

\begin{abstract}
Historically, cardiac amyloidosis (CA) has been considered a rare disease; however, recent research has revealed a considerable number of hidden CA cases in patients with heart failure, especially in those with heart failure with preserved ejection fraction, implying that the actual incidence of CA is probably underestimated. Typically, cardiac involvement signifies a poor prognosis in patients with systemic amyloidosis. However, recent advancements in therapeutic interventions have contributed to improved prognosis in these patients. Apparently, early diagnosis and intervention with effective therapies are imperative, because adequate therapeutic effects are not achieved in patients with late-stage CA. Advanced diagnostic imaging modalities, such as echocardiography, cardiac magnetic resonance imaging, and bone scintigraphy, facilitate a precise and early diagnosis of CA, providing appropriate management to patients with CA. This review aims to outline the clinical features of CA and the recent advancements in noninvasive diagnostic approaches using imaging modalities for CA.
\end{abstract}
Key words Amyloidosis · Heart failure - Cardiomyopathies · Cardiac imaging techniques . Magnetic resonance imaging.

\section{INTRODUCTION}

Amyloidosis implies a spectrum of rare disorders caused by progressive extracellular accumulation of amyloid fibrils derived from misfolded precursor proteins with proteolytic-resistant $\beta$-pleated sheet conformations [1]. Apparently, both the circulating oligomers, which are cytotoxic, and the fibrils, which distort the tissue architecture, result in organ dysfunction. To date, over 30 different precursor proteins implicated in various amyloid diseases, arising as hereditary or nonhereditary and localized or systemic, with different organ involvements and prognoses, have been identified [2]. Cardiac amyloidosis (CA), a progressive infiltrative cardiomyopathy characterized by restrictive cardiomyopathy, is the most severe manifestation of several amyloid types and is typically a major determinant of

(a) This is an Open Access article distributed under the terms of the Creative Commons Attribution Non-Commercial License (http://creativecommons.org/licenses/bync/4.0) which permits unrestricted non-commercial use, distribution, and reproduction in any medium, provided the original work is properly cited. patient outcomes [3]. Amyloid fibrils may deposit within any part of the heart, such as the myocardium, vessels, endocardium, valves, epicardium, and parietal pericardium, resulting in progressive diastolic and systolic dysfunction, congestive heart failure, and conduction diseases, including atrioventricular block with faintness, syncope, sudden death, and occasionally ischemia [4]. For a long time, CA has been considered a rare disease; however, recent research has revealed a considerable number of hidden CA cases in patients with heart failure, especially in those with heart failure with preserved ejection fraction (HF$\mathrm{pEF})$. HFpEF is the common form of heart failure and accounts for $40-50 \%$ of all causes of heart failure. The mortality rate associated with HFpEF is comparable with that associated with heart failure with reduced ejection fraction and has garnered increased attention to HFpEF.

Frequently, CA is misdiagnosed as hypertensive heart disease, and delayed diagnosis results in significant consequences for patients. Reportedly, the average time from initial symp- 
toms to diagnosis is two years and requires consultation with at least five physicians before reaching a definitive diagnosis of CA [5]. Furthermore, the true incidence of CA is probably underestimated. Cardiac involvement usually signifies a poor prognosis in patients with systemic amyloidosis. However, recent advancements in therapeutic interventions have contributed to an improved prognosis in such patients. Based on the context of amyloid fibril precursors, treatment strategies for CA, such as chemotherapy, liver transplantation, and novel drugs, vary significantly. Early diagnosis and intervention with novel effective therapies are imperative, because sufficient therapeutic effects are not achieved in patients with late-stage CA. Thus, this review aims to outline the clinical features of CA and recent advancements in noninvasive diagnostic approaches using imaging modalities for CA.

\section{MAJOR TYPES OF CA}

Although several amyloidogenic proteins cause CA, two types account for more than $95 \%$ of all CA cases: immunoglobulin light-chain (AL) amyloidosis and transthyretin amyloidosis (ATTR) [4]. In the presence or absence of a genetic mutation, ATTR is subdivided into two types as follows: hereditary or mutant-type ATTR (ATTRm) and wild-type ATTR (ATTRwt) (Table 1).

\section{AL amyloidosis}

$\mathrm{AL}$ amyloidosis is caused by a plasma cell dyscrasia that may occur alone or in association with multiple myeloma. In this disease, monoclonal plasma cells secrete an abnormal immunoglobulin that is insoluble in the serum because of its protein misfolding, thereby subsequently depositing in tissues. Typically, AL amyloidosis affects the heart and kidneys, although involvement in other organs also occurs frequently. Apparently, cardiac involvement is the leading prognostic determinant. A study reported that myocardial amyloid deposition causes cardiomyocyte necrosis and interstitial fibrosis [6]. In addition, direct circulating light-chain toxicity has been considered an important cause of cardiac dysfunction due to oxidative stress [7]. Usually, disease onset is reported when patients are nearing their 60s; it occurs commonly in both males and females [5]. Although the presence of serum monoclonal protein and urinary Bence-Jones protein is highly suggestive of AL amyloidosis, even if these biochemical markers are negative, AL amyloidosis cannot be ruled out because of the limited sensitivity of these analyses. Serum free light chain (FLC) analysis represents a significant advancement in laboratory diagnostics. Its greater sensitivity compared with serum monoclonal protein and urinary Bence-Jones protein analysis has heralded the widespread use of FLC analysis for the diagnosis and follow-up of the treatment response in patients with AL amyloidosis. In addition, serum FLC level is a useful prognostic marker in patients with $\mathrm{AL}$ amyloidosis [8]. A study has reported that cardiac AL amyloidosis is a more aggressive disease than ATTR, with a median untreated survival of fewer than six months in patients with heart failure [9]; this high mortality rate without prompt treatment

Table 1. Main types of cardiac amyloidosis

\begin{tabular}{|c|c|c|c|}
\hline Amyloidosis subtype & AL amyloidosis & $\begin{array}{c}\text { ATTRm } \\
\text { (familial amyloid polyneuropathy) }\end{array}$ & $\begin{array}{c}\text { ATTRwt } \\
\text { (senile systemic amyloidosis) }\end{array}$ \\
\hline Precursor protein & $\begin{array}{l}\text { Monoclonal immunoglobulin } \\
\text { light-chains }\end{array}$ & Mutant variants of TTR & Wild-type TTR \\
\hline Acquired/hereditary & Acquired & Hereditary & Acquired, age-related \\
\hline Organ involvement & $\begin{array}{l}\text { Mainly kidney, heart, liver, } \\
\text { peripheral and/or autonomic } \\
\text { nervous system, soft tissue }\end{array}$ & $\begin{array}{l}\text { Mainly heart, peripheral and/or } \\
\text { autonomic neuropathy }\end{array}$ & $\begin{array}{l}\text { Mainly heart, peripheral nervous } \\
\text { system, carpal tunnel syndrome }\end{array}$ \\
\hline Sex & Male $>$ Female & Male $>>>$ Female & Male $=$ Female \\
\hline Mean age & $\begin{array}{l}\text { Depending on underlying } \\
\text { hematologic diseases }\end{array}$ & 30 s and $50 s$ & Late $60 \mathrm{~s}$ \\
\hline Treatment & $\begin{array}{l}\text { Chemotherapy, autologous } \\
\text { peripheral blood stem } \\
\text { cell transplantation }\end{array}$ & $\begin{array}{l}\text { Supportive care, heart/liver } \\
\text { transplantation, TTR stabilizer, } \\
\text { siRNA therapy }\end{array}$ & $\begin{array}{l}\text { Supportive care, TTR stabilizer, } \\
\text { siRNA therapy }\end{array}$ \\
\hline Echocardiography & Wall thickness + & Wall thickness ++ & Wall thickness ++ \\
\hline $\mathrm{CMR}$ & $\begin{array}{l}\text { Subendocardial LGE, dark blood } \\
\text { pool, elevated native T1 and ECV } \\
\text { values }\end{array}$ & $\begin{array}{l}\text { Subendocardial LGE, dark blood } \\
\text { pool, elevated native T1 and ECV } \\
\text { values }\end{array}$ & $\begin{array}{l}\text { Subendocardial LGE, dark blood } \\
\text { pool, elevated native T1 and ECV } \\
\text { values }\end{array}$ \\
\hline Bone scintigraphy & Cardiac uptake- or \pm & Cardiac uptake ++ & Cardiac uptake ++ \\
\hline
\end{tabular}


highlight the importance of early diagnosis of AL amyloidosis. Compared with previous treatments, recent advancements in the chemotherapy of AL amyloidosis, including the use of proteasome inhibitors, have significantly improved the survival rate [10].

\section{ATTRm}

Transthyretin (TTR) is a protein produced by the liver that transports thyroxine (a thyroid hormone) and maintains a tetramer-to-monomer dissociation rate, usually forming folded monomers. These monomers can partially misfold because of abnormal conditions such as point mutations. Evidently, these misfolded monomers are prone to aggregation, forming prefibrillar species and, subsequently, amyloid fibrils. ATTRm, also known as a familial type of amyloid polyneuropathy, is an inherited disease that is primarily caused by production of a mutant TTR. To date, over 130 genetic mutations of TTR have been reported; of them, the leading mutations are replacement of valine by methionine at position 30 (Val30Met) and replacement of valine by isoleucine at position 122 (Val122Ile) [11]. However, the age at disease onset and clinical manifestations are variable and depend on the specific mutation. Although some genotype-phenotype correlations depending on the differences in gene mutations have been reported, ATTRm primarily affects the peripheral/autonomic nerves followed by the heart. Initially, ATTRm was considered to be restricted to an endemic presence in specific areas, especially Japan; however, recent research has highlighted its worldwide presence [12]. The treatment options comprise liver transplantation and new drugs (diflunisal and tafamidis) to stabilize TTR, which are usually effective, thereby slowing disease progression [13]. In particular, early intervention with novel drugs is effective. Furthermore, a study reported that median survival ranges from 10 to 20 years, and that cardiac involvement negatively affects the prognosis of such patients [14].

\section{ATTRwt}

ATTRwt, also known as senile systemic amyloidosis, is caused by infiltration of wild-type TTR in multiple organs, especially the peripheral neurons and heart. Compared with AL amyloidosis or ATTRm, disease onset in ATTRwt is more likely to be delayed, primarily affecting patients aged over 65 years. There is a significant male predominance, with a male:female ratio of 20:1-50:1 [15]. Previous autopsy studies have reported a $25 \%$ prevalence of ATTRwt infiltration in people aged over 80 years [16]. Although not all infiltrations are clinically significant, the incidence of ATTRwt is higher than expected in the elderly. A recent study reported that ATTRwt accounted for $13 \%$ of all HFpEF cases [17]. In clinical practice, however, ATTRwt can often be misdiagnosed because its clinical features resemble those associated with other types of cardiomyopathies such as hypertensive or hypertrophic heart disease. Notably, carpal tunnel syndrome is a relatively specific neurological symptom in ATTRwt and has been reported in $40-50 \%$ of ATTRwt cases. A study revealed that carpal tunnel syndrome is the leading initial symptom of ATTRwt before the onset of cardiac failure [18]. In addition, cardiac infiltration tends to have a less aggressive course in ATTRwt than in AL amyloidosis, with a median survival after diagnosis of 43-47 months [19]. Although an effective therapy for ATTRwt has yet to be delineated by clinical trials, a phase III global clinical trial is currently evaluating the efficacy of the novel drug tafamidis on ATTRwt.

\section{DIAGNOSIS}

$\mathrm{CA}$ is often hard to diagnose because its signs and symptoms are similar to those of more common diseases. However, it is crucial for diagnosis of CA to have a suspicion of CA based on clinical presentation. Important cardiac manifestations of CA are increased left ventricular (LV) wall thickness, HFpEF (also known as diastolic heart failure), atrial fibrillation, bundle branch block, and complete heart block. In addition, extracardiac symptoms that should raise a suspicion of CA include bilateral carpal tunnel syndrome, spinal canal stenosis, rotator cuff tear, peripheral and autonomic neuropathy, proteinuria, chronic diarrhea, weight loss, macroglossia, periorbital purpu$\mathrm{ra}$, and jaw claudication.

The diagnosis of CA starts with visualization of the echocardiogram in combination with the electrocardiogram. The classic hallmark of CA is a combination of low voltage on electrocardiogram (defined as $\leq 1 \mathrm{mV}$ in all precordial leads or $\leq 0.5$ $\mathrm{mV}$ in all limb leads) and increased LV wall thickness on echocardiogram [20]. In addition, subsequent laboratory tests (including those for plasma B-type natriuretic peptide, troponin $\mathrm{T}$ or I, serum monoclonal protein, urinary Bence-Jones protein, and serum FLC), cardiac imaging [cardiac magnetic resonance imaging (CMR) and bone scintigraphy], and tissue biopsy (abdominal fat pad, subcutaneous tissue, gastrointestinal tract, and myocardium) are used to validate the diagnosis of amyloidosis.

Apparently, endomyocardial biopsy is the gold standard for demonstrating cardiac amyloid deposition; however, this procedure is invasive and cannot be performed routinely. Furthermore, the false-negative biopsy interpretation rate is generally high. Thus, CA diagnosis is acceptable even without performing an endomyocardial biopsy if a patient exhibits positive staining for amyloid fibrils in other involved organs and presents with characteristic structural and functional changes compatible with CA in echocardiography, CMR, and bone scintigraphy. However, an endomyocardial biopsy should be considered for the diagnosis of CA in the case of negative Congo-red staining 
in affected organs or abdominal fat.

\section{NONINVASIVE DIAGNOSTIC IMAGING FOR CA}

\section{Echocardiography}

Echocardiography, the standard-of-care testing for all patients with heart failure, is the imaging modality that frequently raises the suspicion of CA. Conventionally, "granular sparkling" or "speckling" appearance of the myocardium was considered one of the features of CA; this sign is observed in other types of cardiomyopathy as well. Although the characteristic myocardial echogenicity was readily detected by conventional echocardiographic imaging, the recent echocardiographic imaging using tissue harmonics decreases the frequency of occurrence of granular sparkling appearance and has a low sensitivity and specificity for diagnosis of CA [21]. In the setting of a non-dilated ventricle, CA is characterized by symmetrically increased biventricular wall thickness (LV wall thickness $>12 \mathrm{~mm}$ and often $\geq 15$ $\mathrm{mm}$ ). Typically, the wall thickness is more prominent in patients with ATTR than in those with AL amyloidosis. The LV ejection fraction is often normal even in the presence of congestive heart failure. Reportedly, diastolic dysfunction is the earliest echocardiographic abnormality, which might precede cardiac symptoms [22]. A study has recommended the ratio of transmitral Doppler early filling velocity (E/e') as a marker for noninvasive estimation of diastolic dysfunction [23]. Recently, two-dimensional speckle-tracking echocardiography has been established as a useful modality to distinguish CA from other types of LV hypertrophy. In addition, as apical sparing of the longitudinal strain, a well-preserved apical strain with significant basal impairment, appeared before reduction of global ejection fraction, this is recognized as a clue to differentiate CA from other types of LV hypertrophy [24].

\section{CMR}

CMR can assess both the structure and function of the heart, uniquely facilitating myocardial tissue characterization. CA is typically assessed using cine CMR and late gadolinium enhancement (LGE) imaging. Myocardial T1 mapping is a novel CMR technique that enables noninvasive detection and quantification of myocardial damage and is becoming popular for the diagnosis of CA. In addition, CMR myocardial strain imaging facilitates the detection of a subclinical disease of CA with the potential for improving patient management.

Apparently, cine CMR imaging is a highly reproducible tool that assesses the myocardial morphology as well as global and regional heart function. Customarily, concentric and symmetric hypertrophy of LV is known to characterize CA. However, recent studies revealed that $42-79 \%$ of patients with CA presented with asymmetric LV hypertrophy, and normal geometry was observed in 3-8\% on cine CMR [25,26] (Fig. 1). Reportedly, LV hypertrophy is more prominent in ATTR than in amyloid AL amyloidosis [27]. In addition, right ventricular involvement with hypertrophy has often been observed. In cardiac functional analysis with cine CMR, traditional markers such as the ejection fraction may be normal even in the late phase of the disease, because it is a poor measure of systolic function in patients with concentric remodeling. Thus, LV stroke volume, which is typically severely reduced, is a better measure of systolic function than is ejection fraction in CA. Although morphological and functional assessment using cine CMR is useful in the diagnosis of CA, these characteristics are nonspecific and differ in their prevalence until the late phases of the disease. In a previous study, the extracardiac findings demonstrated the common presence of pleural and pericardial effusions in $48 \%$ and $35 \%$ of patients with CA, respectively [27]. The presence of ascites, suggestive of right heart failure, is often observed in the later phases of CA. Occasionally, the pres-
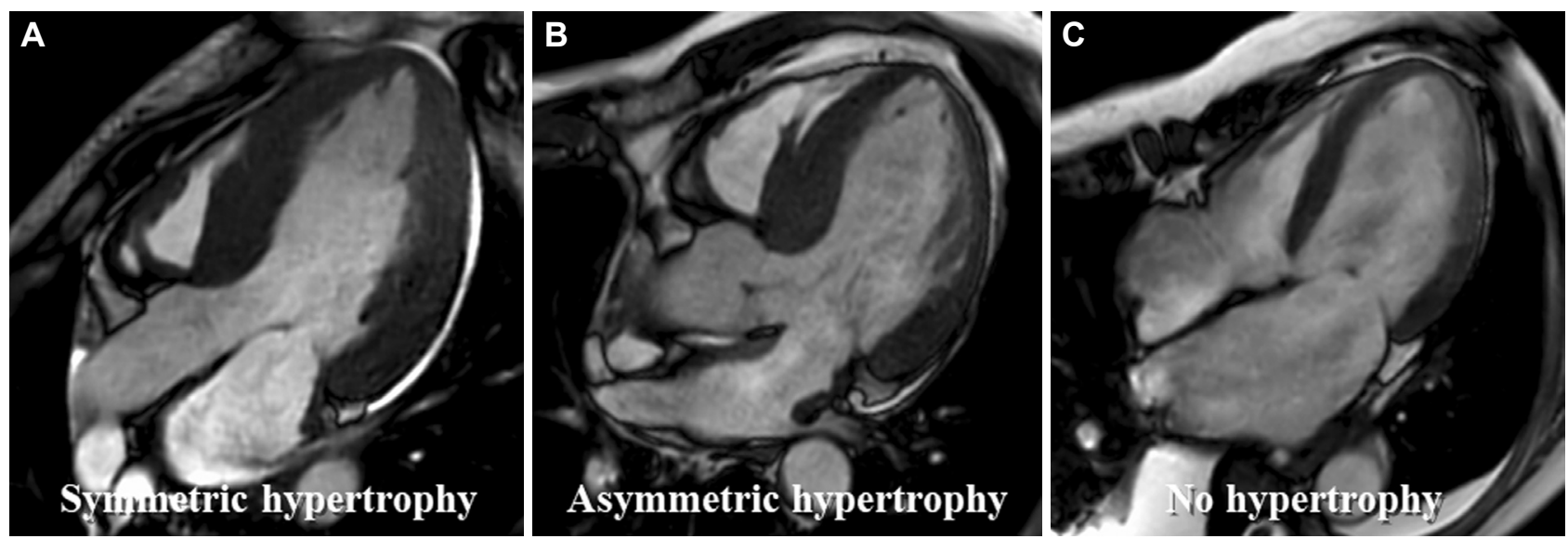

Fig. 1. Cine cardiac magnetic resonance imaging and left ventricular remodeling phenotypes in CA. (A) Symmetric hypertrophy, traditionally considered the characteristic pattern. (B) Asymmetric hypertrophy, recently shown to be highly prevalent in CA. (C) No hypertrophy, a less uncommon finding, but should also be recognized. CA: cardiac amyloidosis. 
ence of increased gas in the bowel or dilated esophagus due to autonomic dysfunction has also been reported.

At present, LGE imaging is the most reliable method for identification of cardiac involvement in amyloidosis [28]. Gadolinium-based contrast agents are intravenously administered, and delayed imaging is performed at least 10 min later to achieve optimum contrast between the normal and infarcted myocardia. Usually, an inversion recovery (IR) sequence is used to null the signal from the normal myocardium. Reportedly, the typical LGE pattern of CA is global subendocardial LGE in a noncoronary artery territory distribution with a dark blood pool [29] (Fig. 2); the finding of a dark blood pool is attributed to the abnormal kinetics of contrast agents in patients with amyloidosis, accounting for a smaller difference between the blood pool and the myocardial intensity. Some studies have demonstrated that a significant correlation of LGE with clinical, morphological, functional, and biochemical markers facilitates the evaluation of the prognosis of CA [30,31]. Reportedly, LGE corresponds to the interstitial amyloid deposition and subendocardial fibrosis caused by ischemia due to capillary obstruction by amyloid deposits $[29,32]$. However, recent studies have revealed a more variable LGE pattern in CA (e.g., diffuse transmural or more localized and patchy LGE) [33]. In addition, it is assumed that LGE findings vary according to cardiac amyloid progression over time [31]. Notably, the following three steps can be identified when amyloid starts accumulating: 1) no evidence of LGE; 2) appearance of subendocardial LGE (typical pattern); and 3) progression to transmural LGE. An atypical LGE pattern can result in the false-negative interpretation of CA. In addition, traditional LGE imaging using the IR method poses technical difficulties, as it was designed for focal myocardial fibrosis, especially myocardial infarction, where substantial regional differences exist in the gadolinium concentrations between the infarct and the re- mote normal myocardium. Diffuse diseases of the myocardium, especially CA, that affect the entire myocardium may have no normal myocardium; this issue in addition to the abnormal kinetics of contrast agents in the blood pool uncover a pitfall of the traditional LGE imaging approach, as the operator defining the optimal myocardial null point may erroneously choose null as abnormal and not the normal myocardium, causing inappropriate inversion time settings. Presumably, these events carry a serious risk of false-negative findings or of obtaining a mirror image of the true pattern, potentially contributing to the described variable LGE patterns [34]. Seemingly, this problem can be reduced by using a phase-sensitive inversion recovery sequence that decreases the need for an optimal null point setting and renders LGE imaging far more accessible and operator independent [31]. Apparently, LGE manifests in the later disease stages, complicating early detection of the disease. Furthermore, the use of gadolinium-based contrast agents is relatively contraindicated in patients with severe renal failure, a relatively common finding in patients with amyloidosis. As LGE is not easy to quantify, it is not reliable for following up changes over time. Supposedly, myocardial T1 mapping, a novel approach for myocardial tissue characterization, can overcome these limitations of LGE imaging.

Myocardial T1 mapping, which evaluates the myocardial T1 relaxation time, is a novel technique that facilitates noninvasive detection and quantification of biologically important processes associated with myocardial edema, fibrosis, protein deposition, and other T1-altering substances such as lipid or iron. Non-contrast T1 mapping, called native T1, reflects myocardial disease involving the myocyte and interstitium without using gadolinium-based contrast agents. Furthermore, contrastenhanced T1 mapping enables calculation of the extracellular volume (ECV) fraction. CA exhibits significantly elevated na-
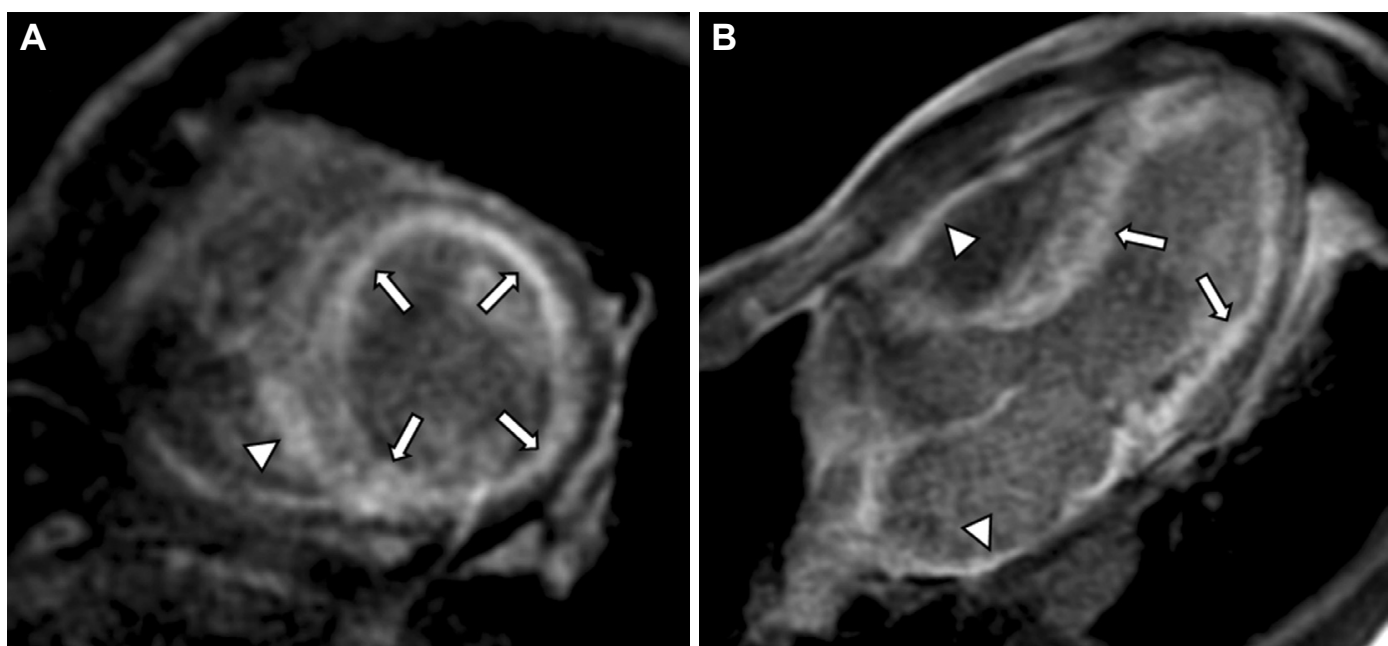

Fig. 2. LGE imaging. Left ventricular short-axis (A) and horizontal long-axis (B) LGE images from a patient with mutated transthyretin amyloidosis showed global subendocardial LGE (arrows) and a dark blood pool, typical of cardiac amyloidosis. Right ventricular and left atrial LGE can also be observed (arrowheads). LGE: late gadolinium enhancement. 
tive T1 and ECV values compared with other cardiac diseases (Fig. 3), exhibiting high diagnostic precision for detection of both AL amyloidosis and ATTR [35]. In addition, native T1 and ECV values are increased even in patients in whom conventional clinical testing and LGE imaging have suggested no cardiac involvement, thereby emphasizing the potential role of native T1 and ECV as early disease markers [36,37]; additionally, they help track various markers of the disease activity, such as cardiac function and blood biomarkers, implying their correlation with severity of CA $[37,38]$. Moreover, the assessment of native T1 and ECV aids in risk-stratification of patients with CA [31,39], which may add incremental value over the existing clinical markers. A recent study highlighted the potential of native $\mathrm{T} 1$ and $\mathrm{ECV}$ as noninvasive imaging markers for disease surveillance monitoring, which can contribute to the management of patients with CA [40].

The handling of myocardial T1 mapping warrants some attention. It is not specific for the disease and quantitatively shows the degree of myocardial damage. In addition, the standard T1 mapping sequence, the look-locker IR (MOLLI) sequence, is sensitive to extreme heart rate values and tends to underestimate the actual myocardial T1 value. Furthermore, myocardial T1 measurements can be altered by numerous factors, such as the magnetic resonance system, acquisition scheme, and magnetic field strength; native $\mathrm{T} 1$ values are higher at 3.0T than at $1.5 \mathrm{~T}$. Thus, it is imperative to set the local normal values for every scanner and method [41]. As discussed earlier, myocardial strain analysis using two-dimensional speckle-tracking echocardiography can successfully detect subtle functional impairment in several diseases and is beneficial for assessing patients with CA. Likewise, myocardial strain analysis using CMR has been established as a technique for assessing regional myocardial function [42]. This facilitates the quantification of lo- cal intramyocardial motion and strain. At present, several CMRbased myocardial strain imaging techniques using various acquisition schemes [e.g., complementary spatial modulation of magnetization (cSPAMM), strain-encoded imaging (SENC), displacement-encoded with stimulated-echo (DENSE), and feature tracking] are available $[42,43]$. Recent research has revealed that CMR-based myocardial strain analysis enables the detection of LGE-positive patients with CA whose prognosis is considered to be poor without using contrast agents. The peak circumferential strain and the variability in the peak circumferential strain time may be correlated with the severity of cardiac amyloid deposition and may be more sensitive than LGE imaging for the detection of early CA [44,45] (Fig. 4).

\section{Bone scintigraphy}

In the differentiation of patients with CA with ATTR from those with cardiac AL amyloidosis and those with non-amyloid heart failure, cardiac uptake on bone scintigraphy with tracers such as ${ }^{99 \mathrm{~m}} \mathrm{Tc}$-pyrophosphate $\left({ }^{99 \mathrm{~m}} \mathrm{Tc}-\mathrm{PYP}\right),{ }^{99 \mathrm{~m}} \mathrm{Tc}-3,3-\mathrm{di}-$ phosphono-1,2-propanodicarboxylic acid ( $\left.{ }^{99 \mathrm{~m}} \mathrm{Tc}-\mathrm{DPD}\right)$, and ${ }^{99 \mathrm{~m}} \mathrm{Tc}$-hydroxymethylene diphosphonate ( $\left.{ }^{99 \mathrm{~m}} \mathrm{Tc}-\mathrm{HMDP}\right)$ has been proven to exhibit high diagnostic performance [46-48] (Fig. 5). In addition, the intense uptake of myocardial nuclear tracers independently predicts the worse survival outcomes in patients with ATTR [48]. On planar images, cardiac retention can be evaluated using a visual scoring method $(0=$ absent cardiac uptake; $1=$ mild uptake less than that of bone; $2=$ moderate uptake equal to that of bone; and 3 =high uptake greater than that of bone) and a quantitative method using the heart-to-contralateral (H:CL) ratio of the total count in a region of interest (ROI) over the heart divided by background count in a copied and mirrored ROI over the contralateral chest to normalize spillover from the ribs.
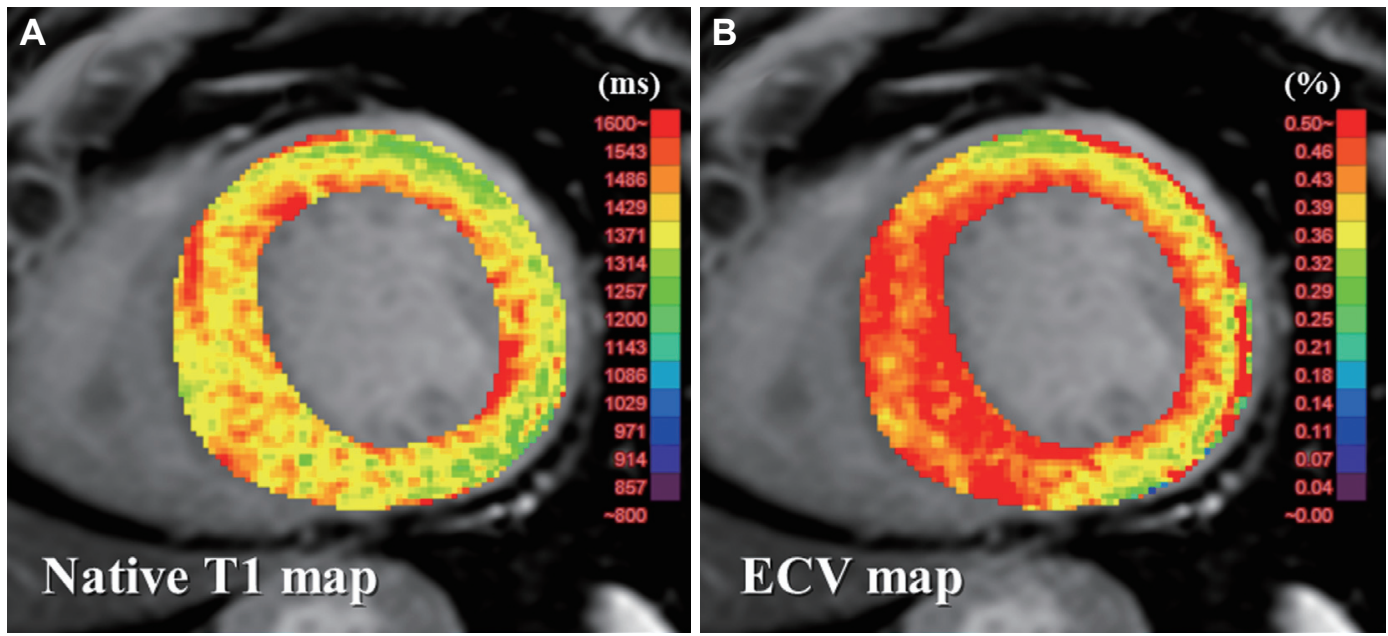

Fig. 3. Myocardial T1 mapping. A patient with cardiac ATTRm. (A) Native T1 map and (B) ECV map. Both native T1 and ECV values are markedly elevated. ECV: extracellular volume. 

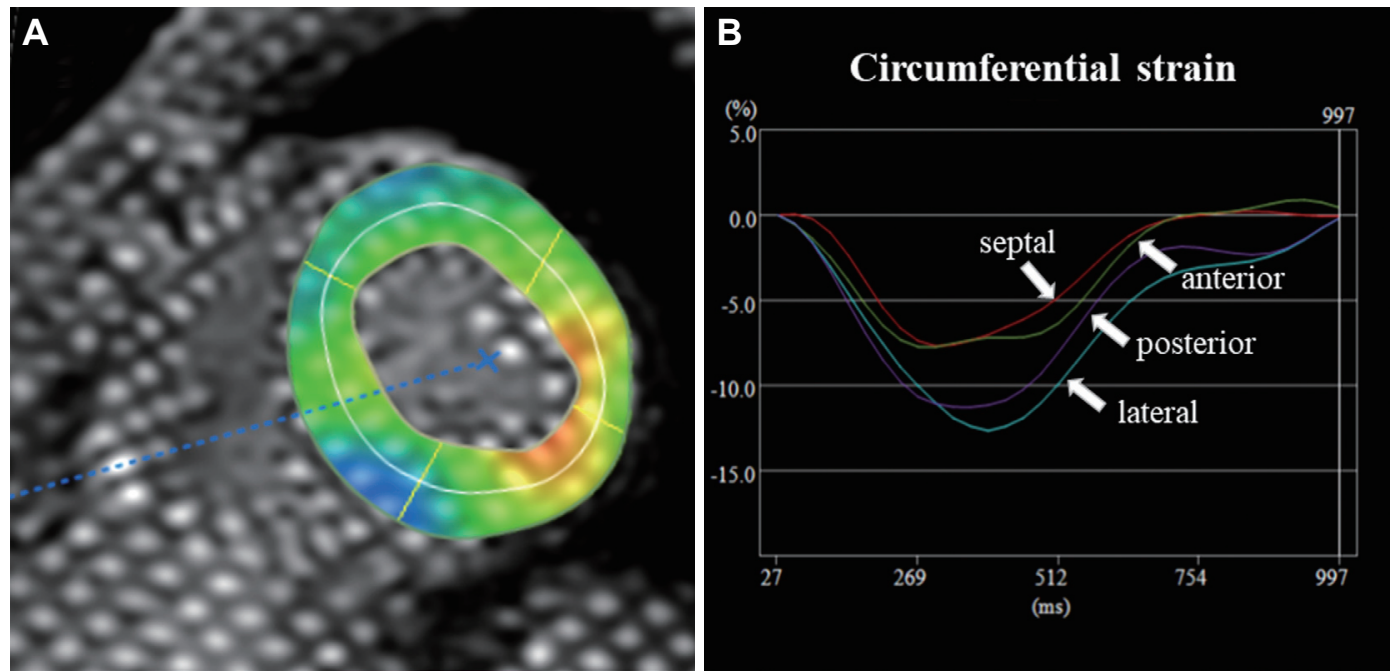

Fig. 4. Cardiac magnetic resonance imaging-based myocardial strain imaging. A patient with cardiac light-chain amyloidosis. Superimposed color-coded strain map at the LV short-axis plane (A) and the circumferential strain time-curves for each LV segment (B). The strain analysis shows non-uniform reduction in the negative peak of circumferential strain. The peak circumferential strain time is delayed and variable. LV: left ventricular.
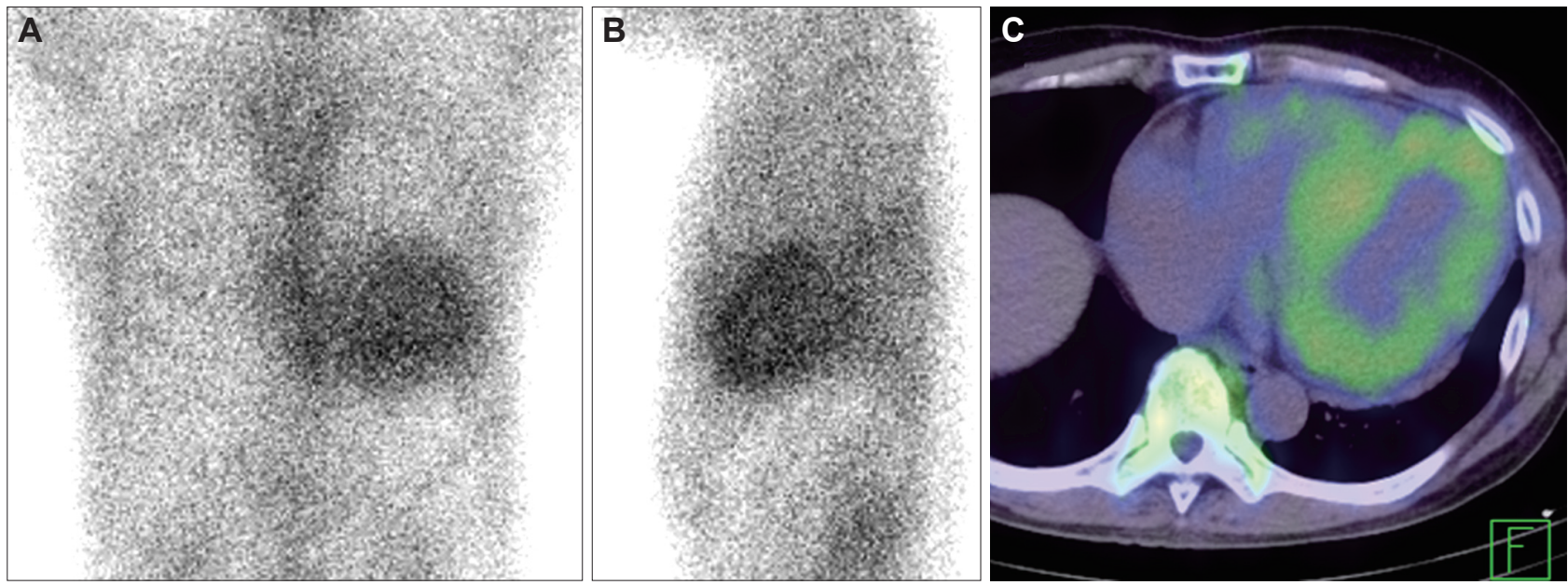

Fig. 5. Bone scintigraphy. A patient with cardiac wild-type transthyretin amyloidosis. ${ }^{99 m}$ Tc-pyrophosphate scintigraphy (A) anterior and (B) lateral planar images exhibit a high myocardial uptake (visual score=3). Single photon emission computed tomography/computed tomography fusion image $(\mathrm{C})$ clearly visualizes the cardiac uptake to be in the myocardium.

A study reported that the myocardial uptake of bone scintigraphy using ${ }^{99 \mathrm{~m}} \mathrm{Tc}-\mathrm{PYP},{ }^{99 \mathrm{~m}} \mathrm{Tc}-\mathrm{DPD}$, and ${ }^{99 \mathrm{~m}} \mathrm{Tc}-\mathrm{HMDP}$ exhibited $>99 \%$ sensitivity and $86 \%$ specificity for detecting cardiac ATTR, with false positives almost exclusively caused by uptake in patients with cardiac AL amyloidosis [46]. In addition, the authors reported that the combined findings of a visual score of 2 or 3 and the absence of a monoclonal protein in the serum or urine had a specificity and positive predictive value of $100 \%$ for cardiac ATTR. Another study reported an H:CL ratio $>1.3$ for a 3-h incubation differentiating cardiac ATTR-CA from cardiac AL amyloidosis with $88 \%$ sensitivity and $86 \%$ specificity [48]. Furthermore, in patients with cardiac ATTR, worse survival was independently predicted by an H:CL ratio $>1.6$ [48]. Although the precise underlying mechanism of the myocardial bone tracer uptake remains unknown in patients with cardiac ATTR, a study has suggested a calcium-mediated mechanism [49]. Apparently, bone tracer physiologically remains in the cardiac blood pool and can result in false-positive scintigrams [50]. Single photon emission computed tomography (SPECT)/computed tomography (CT) fusion imaging can offer better and more useful anatomical information to distinguish myocardial tracer uptake from tracer accumulation in the cardiac blood pool during assessment of cardiac ATTR, and false-positive results can be easily recognized using SPECT/CT fusion imaging.

The role of positron emission tomography imaging in the diagnosis of CA has been reported using new tracers such as ${ }^{11} \mathrm{C}-\mathrm{BF}-227,{ }^{11} \mathrm{C}-\mathrm{PIB}$, and ${ }^{18} \mathrm{~F}$-florbetapir [51]. There can be selective uptake of these tracer in the myocardium of patients with 
CA. Although these new tracers offer the potential to quantify amyloid burden in the heart and to distinguish the different types of amyloid protein, these still require further investigation.

\section{Recent advances in diagnosis of CA with cardiac computed tomography}

Myocardial late iodine enhancement (LIE) imaging with cardiac CT can be an alternative to CMR-LGE imaging because the pharmacokinetics of iodinated contrast materials are analogous to those of gadolinium-containing contrast materials [52].

Dual-energy CT facilitates the simultaneous acquisition of high- and low-energy X-ray datasets. As different materials exhibit different X-ray absorptions at high and low energies, dualenergy CT acquisition can facilitate a higher degree of material characterization than the conventional single-energy acquisition. In clinical practice, the dual-energy post-processing techniques of virtual monochromatic imaging (VMI) and iodine density mapping possess the broadest applicability. A study reported that
VMI can effectively correct beam-hardening artifacts from the chest wall because beam hardening primarily arises from the polychromatic character of the X-rays used in clinical CT scanners [53]. In addition, the contrast resolution of LIE imaging can be improved by taking advantage of the higher iodine contrast in lower-energy images [54-56]. In addition, evaluation of myocardial ECV using iodine density mapping is currently available [57]. Compared with CMR, cardiac CT is more commonly used because of its accessibility, fast acquisition time, ability to perform coronary artery imaging, three-dimensional data, and its suitability for use in patients with mechanical devices such as pacemakers, in patients with patient monitoring devices, or in patients on dialysis that commonly accompanies amyloidosis. Thus, this novel technology of myocardial LIE and ECV analysis using dual-energy cardiac CT is a potentially useful tool for noninvasive assessment of CA (Fig. 6).
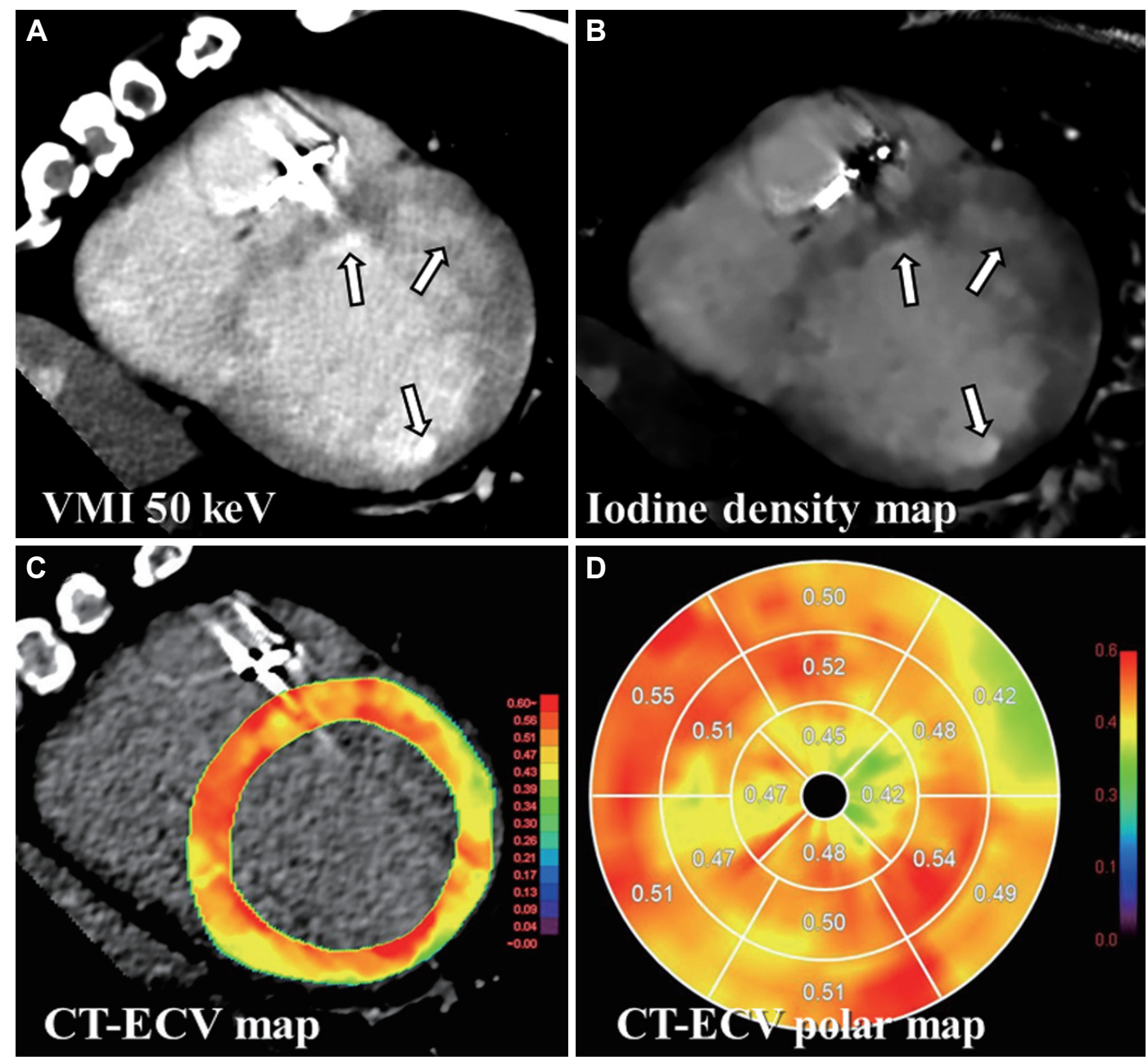

Fig. 6. Dual-energy cardiac CT with LIE and CT-ECV imaging. A patient with cardiac light-chain amyloidosis with an implanted pacemaker. (A) VMl at $50 \mathrm{keV}$ and (B) the iodine density image exhibit multiple diffuse LIEs with an unclear boundary (arrows). (C and D) CT-ECV calculated using iodine density imaging is significantly elevated. CT: computed tomography, LIE: late iodine enhancement, ECV: extracellular volume, VMI, virtual monochromatic imaging. 


\section{CONCLUSION}

Contrary to previously accepted dogma, CA is more common and more prevalent among patients with HFpEF. Recent advancements in disease-targeted strategies signify that CA is treatable or curable. Using the advanced diagnostic imaging modalities discussed in this review, a more precise and early diagnosis of CA can be obtained, along with appropriate management of patients with CA.

\section{Conflicts of Interest}

The authors declare that they have no conflict of interest.

\section{REFERENCES}

1. Wechalekar AD, Gillmore JD, Hawkins PN. Systemic amyloidosis. Lancet (London, England) 2016;387:2641-2654.

2. Sipe JD, Benson MD, Buxbaum JN, Ikeda S, Merlini G, Saraiva MJ, et al. Nomenclature 2014: amyloid fibril proteins and clinical classification of the amyloidosis. Amyloid 2014;21:221-224.

3. Izumiya Y, Takashio S, Oda S, Yamashita Y, Tsujita K. Recent advances in diagnosis and treatment of cardiac amyloidosis. Journal of Cardiology 2018;71:135-143.

4. Maleszewski JJ. Cardiac amyloidosis: pathology, nomenclature, and typing. Cardiovasc Pathol 2015;24:343-350.

5. Lousada I, Comenzo RL, Landau H, Guthrie S, Merlini G. Light chain amyloidosis: patient experience survey from the Amyloidosis Research Consortium. Advanc Ther 2015;32:920-928.

6. Shah KB, Inoue Y, Mehra MR. Amyloidosis and the heart: a comprehensive review. Arch Intern Med 2006;166:1805-1813.

7. Brenner DA, Jain M, Pimentel DR, Wang B, Connors LH, Skinner M, et al. Human amyloidogenic light chains directly impair cardiomyocyte function through an increase in cellular oxidant stress. Circ Res 2004;94: 1008-1010.

8. Dittrich T, Bochtler T, Kimmich C, Becker N, Jauch A, Goldschmidt H, et al. AL amyloidosis patients with low amyloidogenic free light chain levels at first diagnosis have an excellent prognosis. Blood 2017;130:632642.

9. Dubrey SW, Cha K, Anderson J, Chamarthi B, Reisinger J, Skinner M, et al. The clinical features of immunoglobulin light-chain (AL) amyloidosis with heart involvement. QJM 1998;91:141-157.

10. Sperry BW, Ikram A, Hachamovitch R, Valent J, Vranian MN, Phelan D, et al. Efficacy of chemotherapy for light-chain amyloidosis in patients presenting with symptomatic heart failure. J Am Coll Cardiol 2016;67:29412948.

11. Dubrey SW, Hawkins PN, Falk RH. Amyloid diseases of the heart: assessment, diagnosis, and referral. Heart 2011;97:75-84.

12. Plante-Bordeneuve V, Said G. Familial amyloid polyneuropathy. Lancet Neurol 2011;10:1086-1097.

13. Adams D, Cauquil C, Labeyrie C, Beaudonnet G, Algalarrondo V, Theaudin M. TTR kinetic stabilizers and TTR gene silencing: a new era in therapy for familial amyloidotic polyneuropathies. Expert Opin Pharmacother 2016;17:791-802.

14. Yamamoto S, Wilczek HE, Nowak G, Larsson M, Oksanen A, Iwata T, et al. Liver transplantation for familial amyloidotic polyneuropathy (FAP): a single-center experience over 16 years. Am J Transplant 2007;7:25972604.

15. Ruberg FL, Berk JL. Transthyretin (TTR) cardiac amyloidosis. Circulation 2012;126:1286-1300.

16. Cornwell GG 3rd, Murdoch WL, Kyle RA, Westermark P, Pitkanen P. Frequency and distribution of senile cardiovascular amyloid. A clinicopathologic correlation. Am J Med 1983;75:618-623.
17. Gonzalez-Lopez E, Gallego-Delgado M, Guzzo-Merello G, de Haro-Del Moral FJ, Cobo-Marcos M, Robles C, et al. Wild-type transthyretin amyloidosis as a cause of heart failure with preserved ejection fraction. Europ Heart J 2015;36:2585-2594.

18. Nakagawa M, Sekijima Y, Yazaki M, Tojo K, Yoshinaga T, Doden T, et al. Carpal tunnel syndrome: a common initial symptom of systemic wildtype ATTR (ATTRwt) amyloidosis. Amyloid 2016;23:58-63.

19. Connors LH, Sam F, Skinner M, Salinaro F, Sun F, Ruberg FL, et al. Heart failure resulting from age-related cardiac amyloid disease associated with wild-type transthyretin: a prospective, observational cohort study. Circulation 2016;133:282-290.

20. Sperry BW, Vranian MN, Hachamovitch R, Joshi H, McCarthy M, Ikram A, et al. Are classic predictors of voltage valid in cardiac amyloidosis? A contemporary analysis of electrocardiographic findings. Int J Cardiol 2016;214:477-481.

21. Picano E, Pinamonti B, Ferdeghini EM, Landini L, Slavich G, Orlandini A, et al. Two-dimensional echocardiography in myocardial amyloidosis. Echocardiography 1991;8:253-259.

22. Koyama J, Ikeda S, Ikeda U. Echocardiographic assessment of the cardiac amyloidoses. Circ J 2015;79:721-734.

23. Nagueh SF, Smiseth OA, Appleton CP, Byrd BF 3rd, Dokainish H, Edvardsen $\mathrm{T}$, et al. Recommendations for the evaluation of left ventricular diastolic function by echocardiography: an update from the American Society of Echocardiography and the European Association of Cardiovascular Imaging. Eur Heart J Cardiovasc Imaging 2016;29:277-314.

24. Phelan D, Thavendiranathan P, Popovic Z, Collier P, Griffin B, Thomas JD, et al. Application of a parametric display of two-dimensional speckletracking longitudinal strain to improve the etiologic diagnosis of mild to moderate left ventricular hypertrophy. J Am Soc Echocardiogr 2014;27: 888-895.

25. Pozo E, Kanwar A, Deochand R, Castellano JM, Naib T, Pazos-Lopez P, et al. Cardiac magnetic resonance evaluation of left ventricular remodelling distribution in cardiac amyloidosis. Heart 2014;100:1688-1695.

26. Martinez-Naharro A, Treibel TA, Abdel-Gadir A, Bulluck H, Zumbo G, Knight DS, et al. Magnetic resonance in transthyretin cardiac amyloidosis. J Am Coll Cardiol 2017;70:466-477.

27. Dungu JN, Valencia O, Pinney JH, Gibbs SD, Rowczenio D, Gilbertson JA, et al. CMR-based differentiation of AL and ATTR cardiac amyloidosis. JACC Cardiovas Imaging 2014;7:133-142.

28. Maceira AM, Prasad SK, Hawkins PN, Roughton M, Pennell DJ. Cardiovascular magnetic resonance and prognosis in cardiac amyloidosis. J Cardiovasc Magn Reson 2008;10:54.

29. Syed IS, Glockner JF, Feng D, Araoz PA, Martinez MW, Edwards WD, et al. Role of cardiac magnetic resonance imaging in the detection of cardiac amyloidosis. JACC Cardiovas Imaging 2010;3:155-164.

30. Austin BA, Tang WH, Rodriguez ER, Tan C, Flamm SD, Taylor DO, et al. Delayed hyper-enhancement magnetic resonance imaging provides incremental diagnostic and prognostic utility in suspected cardiac amyloidosis. JACC Cardiovas Imaging 2009;2:1369-1377.

31. Fontana M, Pica S, Reant P, Abdel-Gadir A, Treibel TA, Banypersad SM, et al. Prognostic value of late gadolinium enhancement cardiovascular magnetic resonance in cardiac amyloidosis. Circulation 2015;132:15701579.

32. Hashimura H, Ishibashi-Ueda H, Yonemoto Y, Ohta-Ogo K, Matsuyama TA, Ikeda $Y$, et al. Late gadolinium enhancement in cardiac amyloidosis: attributable both to interstitial amyloid deposition and subendocardial fibrosis caused by ischemia. Heart Vessels 2016;31:990-995.

33. Fontana M, Chung R, Hawkins PN, Moon JC. Cardiovascular magnetic resonance for amyloidosis. Heart Fail Rev 2015;20:133-144.

34. Maceira AM, Joshi J, Prasad SK, Moon JC, Perugini E, Harding I, et al. Cardiovascular magnetic resonance in cardiac amyloidosis. Circulation 2005;111:186-193.

35. Fontana M, Banypersad SM, Treibel TA, Maestrini V, Sado DM, White SK, et al. Native T1 mapping in transthyretin amyloidosis. JACC Cardiovasc Imaging 2014;7:157-165. 
36. Banypersad SM, Sado DM, Flett AS, Gibbs SD, Pinney JH, Maestrini V, et al. Quantification of myocardial extracellular volume fraction in systemic AL amyloidosis: an equilibrium contrast cardiovascular magnetic resonance study. Circ Cardiovasc Imaging 2013;6:34-39.

37. Oda S, Utsunomiya D, Morita K, Nakaura T, Yuki H, Kidoh M, et al. Cardiovascular magnetic resonance myocardial T1 mapping to detect and quantify cardiac involvement in familial amyloid polyneuropathy. Eur Radiol 2017;27:4631-4638.

38. Fontana M, Banypersad SM, Treibel TA, Abdel-Gadir A, Maestrini V, Lane T, et al. Differential nyocyte responses in patients with cardiac transthyretin amyloidosis and light-chain amyloidosis: a cardiac MR imaging study. Radiology 2015;277:388-397.

39. Banypersad SM, Fontana M, Maestrini V, Sado DM, Captur G, Petrie A, et al. T1 mapping and survival in systemic light-chain amyloidosis. Eur Heart J 2015;36:244-251.

40. Hur DJ, Dicks DL, Huber S, Mojibian HR, Meadows JL, Seropian SE, et al. Serial native T1 mapping to monitor cardiac response to treatment in light-chain amyloidosis. Circ Cardiovasc Imaging 2016;9:e004770.

41. Messroghli DR, Moon JC, Ferreira VM, Grosse-Wortmann L, He T, Kellman $\mathrm{P}$, et al. Clinical recommendations for cardiovascular magnetic resonance mapping of $\mathrm{T} 1, \mathrm{~T} 2, \mathrm{~T} 2{ }^{*}$ and extracellular volume: a consensus statement by the Society for Cardiovascular Magnetic Resonance (SCMR) endorsed by the European Association for Cardiovascular Imaging (EACVI). J Cardiovasc Magn Reson 2017;19:75.

42. Jeung MY, Germain P, Croisille P, El ghannudi S, Roy C, Gangi A. Myocardial tagging with MR imaging: overview of normal and pathologic findings. Radiographics 2012;32:1381-1398.

43. Aurich M, Keller M, Greiner S, Steen H, Aus dem Siepen F, Riffel J, et al. Left ventricular mechanics assessed by two-dimensional echocardiography and cardiac magnetic resonance imaging: comparison of high-resolution speckle tracking and feature tracking. Eur Heart J Cardiovasc Imaging 2016;17:1370-1378.

44. Oda S, Utsunomiya D, Nakaura T, Yuki H, Kidoh M, Morita K, et al. Identification and assessment of cardiac amyloidosis by myocardial strain analysis of cardiac magnetic resonance imaging. Circ J 2017;81:10141021.

45. Kuetting DL, Homsi R, Sprinkart AM, Luetkens J, Thomas DK, Schild $\mathrm{HH}$, et al. Quantitative assessment of systolic and diastolic function in patients with LGE negative systemic amyloidosis using CMR. Int J Cardiol 2017;232:336-341.
46. Gillmore JD, Maurer MS, Falk RH, Merlini G, Damy T, Dispenzieri A, et al. Nonbiopsy diagnosis of cardiac transthyretin amyloidosis. Circulation 2016;133:2404-2412.

47. Ruberg FL, Miller EJ. Nuclear tracers for transthyretin cardiac amyloidosis: time to bone up? Circ Cardiovasc Imaging 2013;6:162-164.

48. Castano A, Haq M, Narotsky DL, Goldsmith J, Weinberg RL, Morgenstern R, et al. Multicenter study of planar technetium $99 \mathrm{~m}$ pyrophosphate cardiac imaging: predicting survival for patients with ATTR cardiac amyloidosis. JAMA Cardiol 2016;1:880-889.

49. Stats MA, Stone JR. Varying levels of small microcalcifications and macrophages in ATTR and AL cardiac amyloidosis: implications for utilizing nuclear medicine studies to subtype amyloidosis. Cardiovasc Pathol 2016;25:413-417.

50. Marcus ML, Kerber RE. Present status of the $99 \mathrm{~m}$ technetium pyrophosphate infarct scintigram. Circulation 1977;56:335-339.

51. Aljaroudi WA, Desai MY, Tang WH, Phelan D, Cerqueira MD, Jaber WA. Role of imaging in the diagnosis and management of patients with cardiac amyloidosis: state of the art review and focus on emerging nuclear techniques. J Nucl Cardiol 2014;21:271-283.

52. Schuleri KH, George RT, Lardo AC. Applications of cardiac multidetector CT beyond coronary angiography. Nature Rev Cardiol 2009;6:699-710.

53. So A, Hsieh J, Imai Y, Narayanan S, Kramer J, Procknow K, et al. Prospectively ECG-triggered rapid kV-switching dual-energy CT for quantitative imaging of myocardial perfusion. JACC Cardiovasc Imaging 2012;5:829836.

54. Albrecht MH, Scholtz JE, Husers K, Beeres M, Bucher AM, Kaup M, et al. Advanced image-based virtual monoenergetic dual-energy CT angiography of the abdomen: optimization of kiloelectron volt settings to improve image contrast. Eur Radiol 2016;26:1863-1870.

55. Albrecht MH, Scholtz JE, Kraft J, Bauer RW, Kaup M, Dewes P, et al. Assessment of an advanced monoenergetic reconstruction technique in dual-energy computed tomography of head and neck cancer. Eur Radiol 2015;25:2493-2501.

56. Lv P, Lin XZ, Chen K, Gao J. Spectral CT in patients with small HCC: investigation of image quality and diagnostic accuracy. Eur Radiol 2012; 22:2117-2124.

57. Ohta Y, Kitao S, Watanabe T, Mukai N, Kishimoto J, Yamamoto K, et al. Measurement of myocardial extracellular volume fraction from iodine density images using single-source, dual-energy computed tomography: a feasibility study. J Comput Assist Tomogr 2017;41:750-756. 